\title{
Cooperative Visual SLAM Algorithm BASED on Adaptive Covariance Intersection
}

\author{
Fethi DEMIM ${ }^{1, *}$, Abdelkrim NEMRA ${ }^{1}$, Kahina LOUADJ ${ }^{2}$, \\ Abdelghani BOUCHELOUKH ${ }^{3}$, Mustapha HAMERLAIN ${ }^{4}$, \\ Abdelouahab BAZOULA ${ }^{1}$
}

${ }^{1}$ Laboratoire Vehicules Autonomes Intelligents, Ecole Militaire Polytechnique, EMP, Bordj El Bahri, Algiers, Algeria.

${ }^{2}$ Laboratoire d'Informatique de Mathematiques et de Physique pour Agriculture et les Forets, LIMPAF, Universite de Bouira, Algeria.

${ }^{3}$ National Polytechnic School, ENP, El Harrach, Algiers, Algeria.

${ }^{4}$ Center for Development of Advanced Technologies, CDTA, Baba Hassen, Algiers, Algeria.

*Corresponding Author: Fethi DEMIM (email: demifethi@gmail.com)

(Received: 08-March-2018; accepted: 12-Oct-2018; published: 31-Oct-2018)

DOI: http://dx.doi.org/10.25073/jaec.201823.91

\begin{abstract}
Simultaneous localization and mapping (SLAM) is an essential capability for Unmanned Ground Vehicles (UGVs) travelling in unknown environments where globally accurate position data as GPS is not available. It is an important topic in the autonomous mobile robot research. This paper presents an Adaptive Decentralized Cooperative Vision-based SLAM solution for multiple UGVs, using the Adaptive Covariance Intersection (ACI) supported by a stereo vision sensor. In recent years, SLAM problem has gotten a specific consideration, the most commonly used approaches are the EKFSLAM algorithm and the FAST-SLAM algorithm. The primary, which requires an accurate process and an observation model, suffers from the linearization problem. The last mentioned is not suitable for real-time implementation. In our work, the Visual SLAM (VSLAM) problem could be solved based on the Smooth Variable Structure Filter (SVSF) is proposed. This new filter is robust and stable to modelling uncertainties making it suitable for UGV localization and mapping problem. This new strategy retains the near optimal performance of the SVSF when applied to an uncertain system, it has the added benefit of presenting a consider-
\end{abstract}

able improvement in the robustness of the estimation process. All UGVs will add data features sorted by the ACI that estimate position on the global map. This solution gives, as a result, a large reliable map constructed by a group of UGVs plotted on it. This paper presents a Cooperative SVSF-VSLAM algorithm that contributes to solve the Adaptive Cooperative Vision SLAM problem for multiple UGVs. The algorithm was implemented on three mobile robots Pioneer 3-AT, using stereo vision sensors. Simulation results show efficiency and give an advantage to our proposed algorithm, compared to the Cooperative EKF-VSLAM algorithm mainly concerning the noise quality.

\section{Keywords}

Localization, Map Building, Autonomous Navigation, Data Sensor Fusion, Mobile Robot. 


\section{Introduction}

In the last few years, the simultaneous localization and mapping (SLAM) became an important topic of research in the robotics community. Accurate navigation can be achieved by accurate localization within an accurate map. SLAM is the process that enables a mobile robot to localize and build a map of an unknown environment using only observations relative to the most relevant features detected by its sensors. The beauty of the Kalman Filter (KF) comes from the fact that they estimate a fully correlated posterior over feature maps and robot poses. Their weakness lies in the strong assumptions that have to be made on both the robot motion model and the sensor noise. In addition, the EKF-SLAM algorithm only works with feature maps. And it is not always easy to define and extract features in unstructured and outdoor environments. The EKF approximates the SLAM posterior as a high-dimensional Gaussian overall feature in the map and the robot pose. The single hypothesis and quadratic complexity due to the high dimensional Gaussian approximations for states of the robot and landmarks locations makes the off-diagonal elements of the covariance matrix very large. This causes more complexity and cost increase of computation and, in most cases, diverges the filter [10]. In addition, the EKF covariance matrices are quadratic in the size of the map, and updating them requires time quadratic in the number of landmarks [12. Moreover, when a large number of landmarks are present in the environment, the computation becomes almost impossible. Quadratic complexity is a consequence of the Gaussian representation employed by the EKF.

There is another type of filter started to rise and take place in estimation utilizing the principles of the Unscented Kalman Filter (UKF) uses a unique representation of a Gaussian random variable in $N$ dimensions using $2 N+1$ samples, called sigma points. The representation utilizes the properties of the matrix square root and the covariance definitions to select these points in such a way that they have the same covariance as the Gaussian they approximate 20. The UKF-SLAM results comparable to a third-order
Taylor series expansion of the state-model, while EKF are only accurate to a first-order linearization. The Unscented transform approach also has another advantage: noise can be treated in a nonlinear fashion to account for non-Gaussian or non-additive noises. The UKF suffers less from linearization, though it is not exempt. The UKF does not fully recover from poor landmarks, is the same as in the EKF case.

Fast-SLAM is an algorithm which uses the multi-hypothesis data association and logarithmic complexity instead of quadratic. This approach, known as Fast-SLAM utilizes Rao-Black wellised particle filter to solve the SLAM problem efficiently. Using Fast-SLAM algorithm, the posterior estimation will be over the robot's pose and landmarks locations [11. The FastSLAM algorithm has been implemented successfully over thousands of landmarks and compare to EKF-SLAM that can only handle a few hundreds of landmarks, it has appeared with considerable advantages [9, 11]. The Fast-SLAM shares the fancy property with KF approach when it maintains the full posterior but is much faster compared to the classical KF-SLAM. It can be applied for feature-based and grid-based mapping so that it is also suitable for outdoor applications. In practice, for applications where a consistent global map is required and a realtime performance is not necessary (for example the applications focusing on constructing accurate maps), Fast-SLAM is a better choice. However, for applications where only an instantaneous map is required (obstacle avoidance applications). The development of a new algorithm based on the Smooth Variable Structure Filter (SVSF) is proposed for state and parameter estimation which is robust and stable to modelling uncertainties making it suitable for $\mathrm{Au}$ tonomous Unmanned Vehicle localization and mapping problem [14]-[19].

Many algorithms exist today to solve SLAM problems for the single mobile robot, there is still a challenge to perform cooperative SLAM, especially with a robust filter. Cooperative Simultaneous Localization and Mapping (CSLAM) study requires the use of multiple autonomous UGVs [1. They show many advantages, among them we have: increasing the efficiency of the overall system, extending the error tolerance, improving reliability and/or performance, ra- 
pidity, flexibility, and reduction of cost. The interaction of navigational and sensitivity information provides better features of a defined area. Many studies, experiments and researches were made covering this topic. For instance, Stoy in [2] performed simple relative localization between collaborators. In [3] and 4], research treats the problem of CSLAM with the growing uncertainties supported by simulation and experimental validation. Also, entropy minimization [5], and information theory based techniques were developed to solve Cooperative SLAM problems [6].

The GPS denied environment, the expensive cost of sensors; computational efficiency, software/network performance, and modelling errors and uncertainties are crucial key points in solving CSLAM problem. Research is considering cooperative data fusion, sensor mapping in multiple vehicles, navigation scene [7]. In [8] we find the Extended Kalman Filter based solution for some cooperative SLAM and specifically cooperative visual SLAM in [9, 10]. Also in [11, 12] the cooperative Visual SLAM treated based on particle filter estimation scheme. The work presented in [12] is a part of research work done on autonomous navigation for Micro Aerial Vehicle (MAV), with SVSF filter. To solve SLAM for multiple UGVs, we can consider two main families of solutions: Centralized architecture and Decentralized architecture.

In this paper, we presented a development of a new predictor-corrector called the Adaptive Smooth Variable Structure Filter (ASVSF) based on sliding model theory, using covariance matrices to evaluate the uncertainty of the estimation with optimal adaptive smoothing boundary layer vector to solve the visual SLAM Problem [29, 30. It presents stable and robust faculties in modelling uncertainties [13, 14, which is suitable for the localization and mapping problem of a cooperative UGVs. The adaptive SVSF is a robust recursive estimation method that deals efficiently with initial conditions and modelling errors of the odometer/stereo vision system. Previous studies utilized single camera as the best solution for SLAM problem, using SVSF filter, but in this paper, multiple UGVs system based on the Adaptive Decentralized Cooperative architecture is considered using a stereo vision sensor [28]-[31]. The suc- cess of this application depends highly on the accuracy and robustness of the strategy of the Smooth Variable Structure Filter SLAM implementation. The work presented in this paper is organized as follows: Section 2 illustrates the process models of UGV and stereo vision sensor. Section 3 describes the SVSF-SLAM algorithm and the Adaptive SVSF-SLAM algorithm in details. The cooperative SLAM of multiple UGVs with decentralized architecture is described in Section 4. Simulation and discussion are presented in Section 5 and concluding in Section 6.

\section{Process models OF UGV and stereo vision sensor}

\subsection{Process model}

The UGV used in our work is the Pioneer P3AT. The P3-AT is a non-holonomic robot with four wheels [23. According to [15], with this control input and the location of the robot at the previous time step, we can estimate the robot current location by

$$
\begin{aligned}
\left(\begin{array}{c}
X_{r, k+1} \\
Y_{r, k+1} \\
\theta_{r, k+1}
\end{array}\right)= & \left(\begin{array}{c}
X_{r, k}+\Delta T v_{k} \cos \left(\theta_{r, k}\right) \\
Y_{r, k}+\Delta T v_{k} \sin \left(\theta_{r, k}\right) \\
\theta_{r, k}+\Delta T w_{k}
\end{array}\right) \\
& +\left(\begin{array}{c}
\varepsilon_{x_{r}} \\
\varepsilon_{y_{r}} \\
\varepsilon_{\theta_{r}}
\end{array}\right)
\end{aligned}
$$

The robot evolution model reflects the relationship between the robot previous states $X_{R, k}$ and its current state $X_{R, k+1}$. In SLAM, the system state vector has a position of the $\mathrm{UGV}\left(X_{R}\right)$. It is represented by $X_{R}=\left[X_{r}, Y_{r}, \theta_{r}\right]^{T} \in \mathbf{R}^{3}$, and we call a collection of $M$ features a map such that $L=\left[L_{1}, \ldots, L_{M}\right]^{T} \in \mathbf{R}^{3 M}, \Delta T$ is the sample period, $\varepsilon_{x_{r}, y_{r}, \theta_{r}}$ are the noise that arise from the encoder and wheels slipping, etc. In this work, we will use a point feature such that for the $i^{t h}$ feature: $L_{i}=\left[x_{i}, y_{i}, z i\right]^{T}$. Where $x, y$ and $z$ are the coordinates of the point in a global frame of reference. We can write equation 1 as follow 


$$
X_{R, k+1}=f\left(X_{R, k}, U_{k}, k\right)+\varepsilon_{x_{r}, y_{r}, \theta_{r}}
$$

\subsection{Stereo vision sensor model}

The perspective camera model includes intrinsic and extrinsic parameters. This model ensures the geometric transformation between camera/image and world/camera reference frames respectively 22 .

\section{- Observation Point based Model}

In order to perform the SLAM, the robot needs to be able to select and track the landmarks in its environment to localize itself. In this paper, we opt for a point landmark in $3 D$ space. To compute the relative measurement of the landmarks obtained from the images acquired from the stereo vision sensor (Fig. 1).

For the simulation the SVSF visual SLAM

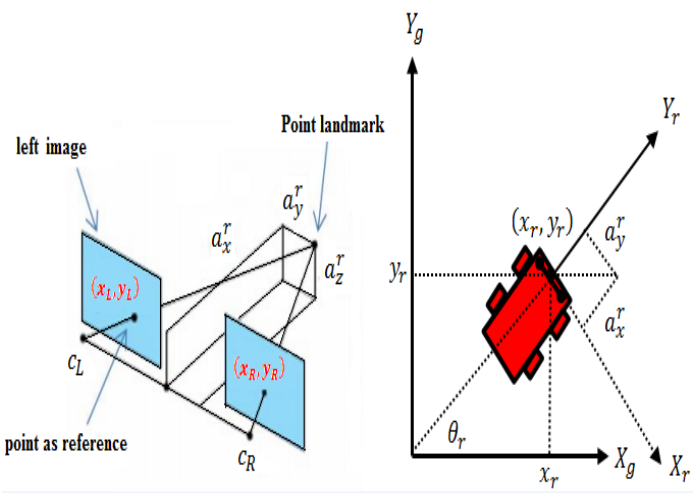

Fig. 1: Observation system geometry.

algorithm, we use theoretical data sets (a set of $3 \mathrm{D}$ points) previously generated instead of using real data. During the robot motion, the point landmarks included in the vision sensor field are detected in $3 D$ space [16. As said previously, the stereo vision sensor provides relative measurement $Z=\left(L_{x}^{r}, L_{y}^{r}, L_{z}^{r}\right)^{T}$ of the landmarks with respect to the robot frame, this measurement (observation) will be noted $Z$. The model representing the robot frame coordinates of an individual landmark, according to its global frame coordinates $L^{g}=\left(L_{x}^{g}, L_{y}^{g}, L_{z}^{g}\right)^{T}$ and the robot configuration $R=\left(x_{r}, y_{r}, 0\right)^{T}$ is called the direct model observation and will be noted 16

$$
\begin{gathered}
Z=h\left(R, L^{g}\right)+\varepsilon_{x, y, z} \\
Z=M_{G R}\left[\begin{array}{c}
L_{x}^{g}-x_{r} \\
L_{y}^{g}-y_{r} \\
L_{z}^{g}-0
\end{array}\right]+\left[\begin{array}{c}
\varepsilon_{x} \\
\varepsilon_{y} \\
\varepsilon_{z}
\end{array}\right]
\end{gathered}
$$

where the global to the robot projection matrix $M_{G R}$ is denoted by

$$
M_{G R}=\left[\begin{array}{ccc}
\left(\cos \left(\theta_{r}\right)\right. & \sin \left(\theta_{r}\right) & 0 \\
-\sin \left(\theta_{r}\right) & \cos \left(\theta_{r}\right) & 0 \\
0 & 0 & 1
\end{array}\right]
$$

and $\varepsilon_{x, y, z}$ presents the measurement noise.

\section{- Inverse observation Point based Model}

The new observed landmark must be initialized previously to be added to the state vector 17 . The initialization process is, in fact, the best estimation of the landmark position, and it is a fundamental point to SLAM implementation. The observation model stated in (4) gives three equations for three dimension variable $L^{g}$. The 3 D coordinates of a new landmark $\left(L_{x}^{g}, L_{y}^{g}, L_{z}^{g}\right)^{T}$ with respect to the global framework are initialized by solving (3) as follows

$$
\begin{gathered}
L^{g}=h^{-1}(R, Z) \\
L^{g}=\left(M_{G R}\right)^{-1} Z+R
\end{gathered}
$$

\section{Visual Adaptive SVSF-SLAM algorithm}

In 2007 , the smooth variable structure filter was introduced. This filter is based on the sliding mode control and estimation techniques and is formulated in a predictor-corrector fashion [15, 18. The estimation process may be summarized by (8) to (13), and is repeated iteratively. An a priori state estimate is calculated using an estimated model of the system [18, 27]. The correct term calculated in (10) is then used in (13) to find the posteriori state estimate. Two 
critical variables in this process are the priori and a posteriori output error estimate, defined by (11) and (12) respectively [18, 25 .

$$
\begin{gathered}
\hat{X}_{k+1}=f\left(\hat{X}_{k}, U_{k}\right) \\
\hat{Z}_{k+1}=h\left(\hat{X}_{k}\right)
\end{gathered}
$$

The gain is computed using the priori, the posteriori measurement error, the smoothing boundary layer widths $\varphi$, convergence rate $\gamma$ and measurement matrix $H_{k+1}$ as follows [15, 26]:

$$
\begin{array}{r}
K_{k+1}^{S V S F}=\hat{H}_{k+1}^{+} \operatorname{diag}\left[\left(\left|e_{z_{k+1 / k}}\right| A b s+\gamma\left|e_{z_{k / k}}\right| A b s\right)\right. \\
\left.{ }^{\circ} \operatorname{Sat}\left(\bar{\varphi}^{-1} e_{z_{k+1 / k}}\right)\right]\left[\operatorname{diag}\left(e_{z_{k+1 / k}}\right)\right]^{-1}
\end{array}
$$

where

- $\circ$ represents "Schur" multiplication element-by-element;

- + refers to the pseudo inverse of a matrix;

- $H_{k+1, j}=h_{k+1, j}\left(F_{X, i}\right)$ is the derivative of $h$ with respect to the state vector $X_{k+1}$, we note that $h$ depends only of the robot pose $R_{k+1}$ and the location of the $i^{t h}$ landmark, where $i$ is the index of the observed landmark at time $k$ and $j$ is the index of an individual landmark observation in $h_{k+1, j}$. $F_{X, i}$ is calculated in [16].

- $\bar{\varphi}^{-1}$ is a diagonal matrix constructed from the smoothing boundary layer vector $\varphi$, such that

$$
\bar{\varphi}^{-1}=[\operatorname{diag}(\varphi)]^{-1}=\left(\begin{array}{ccc}
\frac{1}{\varphi_{1}} & 0 & 0 \\
0 & \ddots & 0 \\
0 & 0 & \frac{1}{\varphi_{M_{i}}}
\end{array}\right),
$$

with $M_{i}$ represents the number of measurements.

- $\operatorname{Sat}\left(\bar{\varphi}^{-1} e_{z_{k+1}}\right)$ represents the saturation function [23, 28,

$$
\begin{gathered}
e_{z_{k+1 / k}}=Z_{k+1}-\hat{Z}_{k+1 / k} \\
e_{z_{k+1 / k+1}}=Z_{k+1}-\hat{Z}_{k+1 / k+1}
\end{gathered}
$$

The update of the state estimates can be calculated as follows

$$
\hat{X}_{k+1 / k+1}=\hat{X}_{k+1 / k}+K_{k+1}^{S V S F} \hat{e}_{z_{k+1 / k}}
$$

SLAM is the problem of constructing a model of the environment being traversed with on board sensors, while at the same time maintaining an estimate of the vehicle location within the model [15, 20. As an alternative approach, there is a novel filter, known as the Adaptive smooth variable structure filter (ASVSF). This research focused on advancing the development and implementation of the Adaptive SVSFVSLAM algorithm using matrix covariance to evaluate the uncertainty of estimating with optimal smoothing boundary layer vector.

In this section we investigate the ASVSFVSLAM proposed algorithm as a new approach. We will show the nonlinear ASVSF which is necessary to solve our Unmanned Ground Vehicle SLAM problem.

The initial conditions used by the ASVSFVSLAM algorithm were the same as those used by the EKF/SVSF-SLAM algorithm. The Adaptive SVSF-VSLAM algorithm can be described as follows:

\section{- Initialization}

The process estimation needs the initialization of the original pose $\hat{X}(0)$ of the coordinate system and covariance matrix $P(0)$. The posteriori measurement error vector $e_{0}^{Z}$ can be is initialized arbitrary in the ASVSFVSLAM algorithm.

$\bar{X}_{0}=\left[\bar{R}_{0}, \bar{L}_{1}^{f, 0}, \ldots, \bar{L}_{M_{0}}^{f, 0}\right]^{T}$ and $e_{0 / 0}^{Z}=\left[e_{0}^{Z_{1}} \ldots e_{0}^{Z_{M_{0}}}\right]^{T}$

where $\hat{X}(0)=[0,0,0]^{T}$ is the initial pose of the UGV, $P_{0}$ is the covariance matrix and $N_{0}$ is the number of initial feature.

$Z_{k}=\left[Z_{1}, Z_{2}, \ldots, Z_{M}\right]^{T}$ be a set of system measurements.

\section{- Prediction}

The prediction stage is a process, which deals with vehicle motion based on incremental dead reckoning estimates and increases the uncertainty of the vehicle pose estimate. The state vector is augmented with a control input $U_{k}$. We consider the following process for the ASVSF estimation strategy, as applied to a nonlinear system. The predicted state estimates $X_{k+1 / k}$ and the predicted covariance matrix $P_{k+1 / k}$ are first calculated as follows: 
$1-X_{k+1}=f\left(\hat{X}_{k}, \hat{U}_{k}\right)=f\left(R_{k+1}, L_{i}^{f, k+1}\right)$

$2-P_{k+1 / k}=\nabla F_{X} P_{k / k} \nabla F_{X}{ }^{T}+$ $\nabla F_{U} Q_{k} \nabla F_{U}^{T}$

where $\nabla F_{X}$ and $\nabla F_{U}$ be the Jacobian matrices of $f($.$) with respect to X_{k+1}$ evaluated at an elsewhere specified point, denoted by

$\nabla F_{X}=\left[\begin{array}{cccc}J_{1} & 0 & \ldots & 0 \\ 0 & 1 & \ldots & 0 \\ \vdots & \vdots & \ddots & \vdots \\ 0 & 0 & \ldots & 1\end{array}\right], \quad$ and
$\nabla F_{U}=\left[\begin{array}{c}J_{2} \\ 0 \\ \vdots \\ 0\end{array}\right]$ where

$$
\begin{gathered}
J_{1}=\left[\frac{\partial f(.)}{\partial x_{r}} ; \frac{\partial f(.)}{\partial y_{r}} ; \frac{\partial f(.)}{\partial \theta_{r}}\right] \\
=\left[\begin{array}{ccc}
1 & 0 & -v \sin \left(\theta_{r}\right) \Delta T \\
0 & 1 & v \cos \left(\theta_{r}\right) \Delta T \\
0 & 0 & 1
\end{array}\right] \\
J_{2}=\left[\frac{\partial f(.)}{\partial v} ; \frac{\partial f(.)}{\partial w}\right]=\left[\begin{array}{cc}
\cos \left(\theta_{r}\right) \Delta T & 0 \\
\sin \left(\theta_{r}\right) \Delta T & 0 \\
0 & 0
\end{array}\right] \Delta T \\
Q_{k}=\left[\begin{array}{ccc}
\sigma_{v}^{2} & 0 \\
0 & \sigma_{w}^{2}
\end{array}\right], \\
R_{k}=\left[\begin{array}{ccc}
\sigma_{x_{i}}^{2} & 0 & 0 \\
0 & y_{i}^{2} & 0 \\
0 & 0 & z_{i}^{2}
\end{array}\right]
\end{gathered}
$$

\section{- Data association and update}

The feature already stored in the map is observed by a vision sensor with the measurement $Z_{k+1}$.

3 -For all features observations $Z_{k+1, j}$. $4-\hat{Z}_{k+1, i}=h\left(\hat{X}_{k+1}\right)=h\left(\hat{R}_{k+1}, L_{i}^{f, k+1}\right)$ 5 - if the the landmark $j$ is seen before (correspondence is founded).

The posteriori measurements error vector $\hat{e}_{z_{k+1, i}} \in R^{3 * 1}$ may be calculated by $6-\hat{e}_{z_{k+1, i}}=Z_{k+1, j}-\hat{Z}_{k+1, i}$ $P_{k+1 / k}^{\bar{\varphi}} \in R^{6 * 6}$ matrix that is extracted from $P_{k+1 / k}$ is calculated by

$7-P_{k+1 / k}^{\bar{\varphi}}=\left[\begin{array}{cc}P_{R} & P_{L_{i}^{f, k+1}, R} \\ P_{R, L_{i}^{f, k+1}} & P_{L_{i}^{f, k+1}}\end{array}\right]$ where $P_{R} \in R^{3 * 3}, P_{L_{i}^{f, k+1}, R} \in 0^{3 * 3}$, $P_{R, L_{i}^{f, k+1}} \in 0^{3 * 3}$ and $P_{L_{i}^{f, k+1}} \in R^{3 * 3}$.

The array width of the boundary layer $\bar{\varphi}_{k+1}^{\text {opt }} \in R^{2 * 2}$ may be calculated by

$8-\bar{\varphi}_{k+1}^{o p t}=\left(\nabla h_{k+1} P_{k+1 / k}^{\bar{\varphi}}\left(\nabla h_{k+1}\right)^{T}+\right.$ $\left.R_{k}\right)\left(\nabla h_{k+1}\right.$

$\left.P_{k+1 / k}^{\bar{\varphi}}\left(\nabla h_{k+1}\right)^{T}\right)^{-1}\left[\operatorname{diag}\left(\left|\hat{e}_{z_{k+1, i}}\right|_{A b s}+\right.\right.$ $\left.\left.\gamma\left|\hat{e}_{z_{k, i}}\right| A b s\right)\right]$

where

$\nabla h_{k+1}=\left[\frac{\partial h\left(\hat{X}_{k+1 / k}\right)}{\partial X_{R}}, \frac{\partial h\left(\hat{X}_{k+1 / k}\right)}{\partial L_{i}^{f, k+1}}\right]$

Use the $\bar{\varphi}^{\text {opt }}$ to calculate SVSF gain $K_{k+1}^{A S V S F} \in R^{6 * 3}$

$9^{-} \quad K_{k+1}^{A S V S F}=$

$\left(H_{k+1, j}\right)^{+} \operatorname{diag}\left[\left(\left|e_{z_{k+1, i}}\right|_{A b s}\right.\right.$

$\left.\gamma\left|e_{z_{k, i}}\right| A b s\right)^{\circ} \operatorname{Sat}\left(\left(\bar{\varphi}_{k+1}^{o p t}\right)^{-1}\right.$

$\left.\left.\hat{e}_{z_{k+1, i}}\right)\right]\left[\operatorname{diag}\left(\hat{e}_{z_{k+1, i}}\right)\right]^{-1}$

$\left(H_{k+1, j}\right)^{+}=\left(F_{X, i}\right)^{T}\left(h_{k+1, j}\right)^{+}$: used (21) to calculate $\left(H_{k+1, j}\right)^{+}$Note that the matrix $h_{k+1, j}=\frac{\partial h\left(\hat{X}_{k+1 / k}\right)}{X_{k+1}}$ is the Jacobian of with respect to $R_{k+1}$ and $L_{i}^{f, k+1}$.

The gain vector $K_{k+1}^{A S V S F}$ is used to formulate a posteriori state estimate and the update of the state estimate can be calculated as follows

$10-\hat{X}_{k+1 / k+1}=\hat{X}_{k+1 / k}+K_{k+1}^{A S V S F} \hat{e}_{z_{k+1, i}}$ Update $P_{k+1 / k+1} \in R^{6 * 6}$

$11-P_{k+1 / k+1}=\quad\left(I_{6 * 6}-\right.$
$\left.K_{k+1}^{A S V S F} \nabla h_{k+1}\right) P_{k+1 / k}^{\varphi}$
$\left(I_{6 * 6}-K_{k+1}^{A S V S F} \nabla h_{k+1}\right)^{T}+$
$K_{k+1}^{A S V S F} R_{k}\left(K_{k+1}^{A S V F}\right)^{T}$

The priori measurements error vector $e_{z_{k+1 / k}} \in R^{3 * 1}$ may be calculated by

$12-e_{z_{k+1, i}}=Z_{k+1, j}-h\left(\hat{R}_{k+1}, L_{i}^{f, k+1}\right)$

13-End if

14-End For

$15-\hat{X}_{k+1 / k+1}=\hat{X}_{k+1 / k}, \quad P_{k+1 / k+1}=$ $P_{k+1 / k}$

\section{- Map Management}

As the environment is explored, new features are observed and should be added to the stored map. In this case, the state vector and the output error estimate matrix are calculated from the new observation 21, 23. 


\section{Decentralized Cooperative Visual SLAM}

Communication is a central issue for multiple vehicle systems because it determines the possible modes of interaction among vehicles, as well as their ability to successfully build a world model [20]. It is exciting to know how the mul-

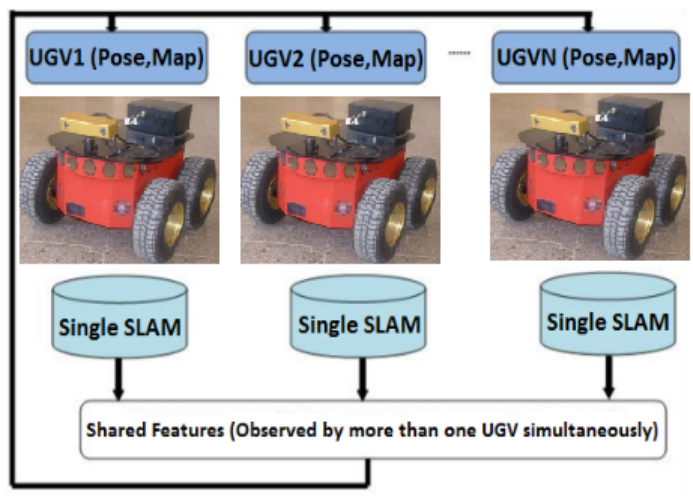

Fig. 2: Decentralized Cooperative SLAM architecture.

tiple UGVs can help each other to solve the Cooperative vision SLAM problem. Figure 2 shows the architecture of the Decentralized Cooperative SLAM (DC-SLAM). Each UGV when shared features are observed in their positions and uncertainties are updated using distributed estimation. The proposed DC-SLAM will be mainly based on the third form of interaction which is via explicit communication. The most restrictive constraint for explicit communication is the limited amount of data to communicate between UGVs. As a result, the collective data to share should be selected carefully in order to maximize the gain with a minimum communication [20. Assume we have $N$ UGVs $\left(U G V_{1}, U G V_{2}, \ldots, U G V_{N}\right)$. At $t=t_{i}$ each $U G V_{i}$ observes $M_{i}$ feature feat ${ }^{i}\left(x^{i}, y^{i}, z^{i}\right)$. Our strategy is then to detect possible shared region which is as follows (Fig. 3): For each $U G V_{i}$ we calculate the mean $\left(\mu^{i}\right)$ and the standard deviation $\left(\sigma^{i}\right)$ of the observed features $\left(\right.$ feat $\left.^{i}\right)$. Then, each set of observed features will be approximated by an ellipsoid $\left(\xi^{i}\right)$ centered at $\left(\mu^{i}\right)$ with axes $\left(\sigma^{i}\right)$. Therefore $U G V_{i}$ and $U G V_{j}$ will

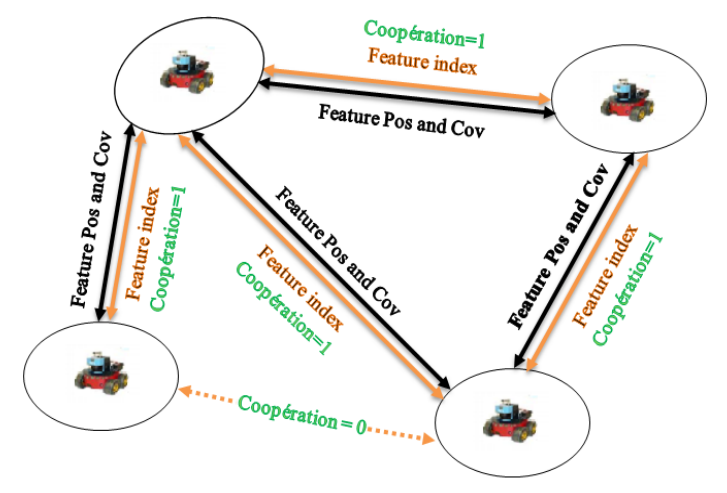

Fig. 3: ACI Decentralized Cooperative VSLAM strategy.

cooperate (Cooperation $(i, j)=1$ ) if and only if: $\xi^{i} \cap \xi^{j} \neq \emptyset[20$. The proposed DC-SLAM strategy is defined as follows: assume we have three $\mathrm{UGVs}\left(U G V_{i}, U G V_{j}\right.$ and $\left.U G V_{k}\right)$ observing a number of features $\left(N_{i}, N_{i}\right.$ and $\left.N_{k}\right)$ respectively. Each observed feature has a description or an index. The Adaptive Covariance Intersection (ACI) approach is used to estimate the position and covariance of shared features before adding them to the global map [20, 24].

\section{SIMULATION, EXPERIMENTS AND DISCUSSION}

In order to verify the efficiency of the Adaptive Cooperative SVSF-SLAM comparing with the cooperative EKF-SLAM. Suppose that the observation noise obeys the mixture Gauss distribution as $\sigma_{x}=\sigma_{y}=10^{-3} \mathrm{~m}, \sigma_{\theta}=$ $10^{-4} \mathrm{rad}$. The convergence rate matrix $\gamma=$ $\operatorname{diag}(0.8,0.8,0.8)$ and the width of the smoothing boundary layer vector used is $\varphi=$ $[21 ; 21 ; 15]$. The sampling rates used for each filter and sensors used in this study are as follows $f_{\text {Odom }}=f_{\text {Camera }}=f_{E K F}=f_{S V S F}=$ $f_{A S V S F}=10 \mathrm{~Hz}$.

We suppose that the observation noise obeys the mixture Gauss distribution as [16] $\varepsilon_{x_{r}, y_{r}, \theta_{r}} \sim$ $0.5 N\left(0, R_{1}\right)+0.5 N\left(0, R_{2}\right)$ and the motion noise obeys the Gauss distribution as $N\left(0, Q_{k}\right)$, 


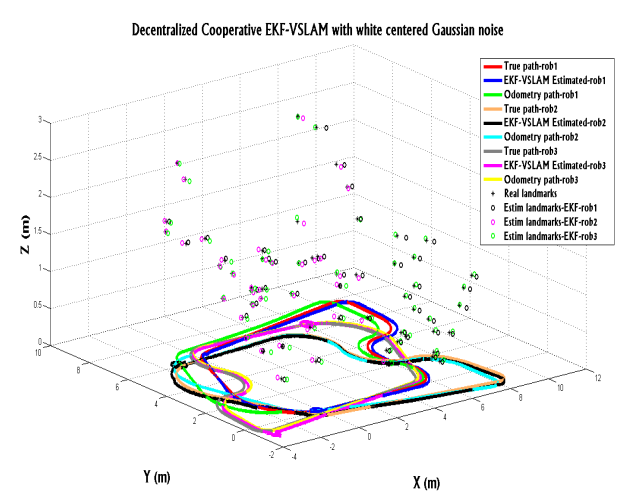

Fig. 4: Visual EKF-SLAM simulation results with white centered Gaussian noise.

$Q_{k}=\left[\begin{array}{cc}0.02 & 0 \\ 0 & 0.01\end{array}\right]$ rate matrix $\gamma=$ $\operatorname{diag}(0.8,0.8,0.8)$

\section{- Test 1 : with white centered Gaussian} noise

In the first experiment, we assume a white centered Gaussian noise, for process and observation model where $\sigma_{v}=0.02 \mathrm{~m} / \mathrm{s}, \sigma_{w}=0.01$ $\mathrm{rad} / \mathrm{s}, \sigma_{x}=0.1 \mathrm{~m}, \sigma_{y}=0.1 \mathrm{~m}$ and $\sigma_{z}=0.1 \mathrm{~m}$.

$$
\begin{aligned}
Q_{k} & =\left(\begin{array}{cc}
0.02 & 0 \\
0 & 0.02
\end{array}\right), \\
R_{k} & =\left(\begin{array}{ccc}
0.01 & 0 & 0 \\
0 & 0.01 & 0 \\
0 & 0 & 0.01
\end{array}\right)
\end{aligned}
$$

Figures $4,5,6$ and 7 present the results of a comparison of the estimated position and errors of the UGVs given by the EKF-VSLAM and ASVSF-VSLAM. As can be seen from these figures when the process and observation noises are centered white Gaussian noises the cooperative EKF-VSLAM performs much better than the cooperative ASVSF-VSLAM. The UGVs poses errors are shown in Fig. 6. This figure confirms the previous conclusion, and it is clear that the cooperative EKF-VSLAM algorithm requires zero-mean white noise, otherwise, the errors pose decrease significantly following $x, y$ and $\theta$ comparing to cooperative ASVSFVSLAM algorithm. Moreover, from the Fig. 7

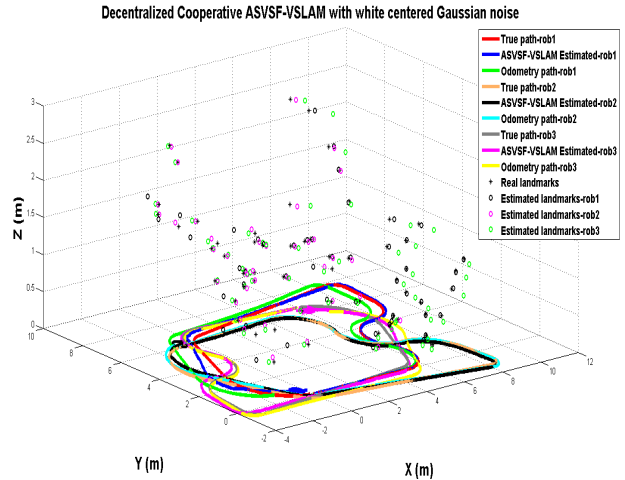

Fig. 5: Visual ASVSF-SLAM simulation results with white centered Gaussian noise.
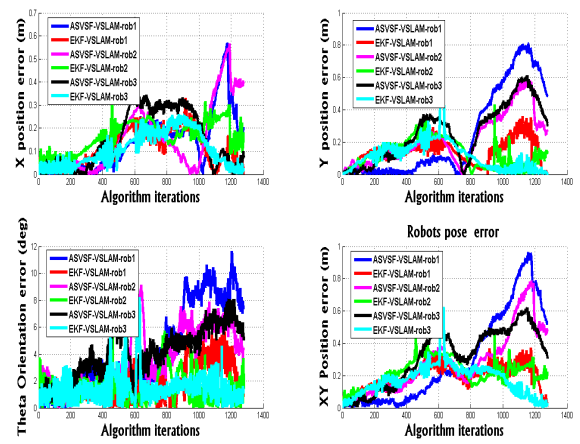

Fig. 6: Position errors of cooperative Visual SLAM with white centered Gaussian noise

we can observe that at many loop closing are detected when the UGVs observes themselves, also when the UGVs observes features already observed previously.

\section{- Test 2 : with non-centered Gaussian} noise

In this experiment we assume a white noise with bias, for process and observation model where $\sigma_{v}=0.02 \mathrm{~m} / \mathrm{s}, \sigma_{w}=0.01 \mathrm{rad} / \mathrm{s}$, $\sigma_{x}=0.1 \mathrm{~m}, \sigma_{y}=\sigma_{z}=0.1 \mathrm{~m}$.

$$
\begin{aligned}
Q_{k} & =\left(\begin{array}{cc}
0.02 & 0 \\
0 & 0.02
\end{array}\right), \\
R_{k} & =\left(\begin{array}{ccc}
0.01 & 0 & 0 \\
0 & 0.01 & 0 \\
0 & 0 & 0.01
\end{array}\right)
\end{aligned}
$$




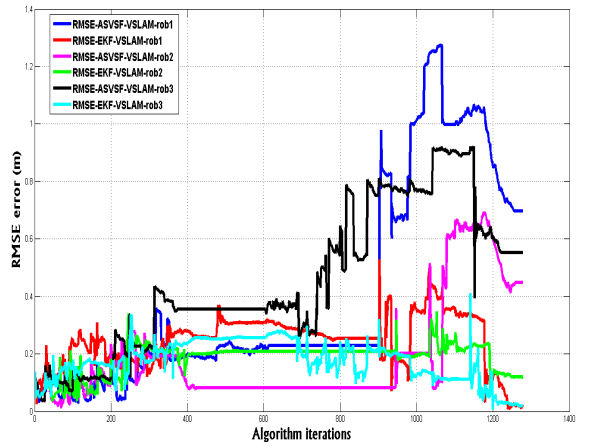

Fig. 7: RMSE Results with white centered Gaussian noise.

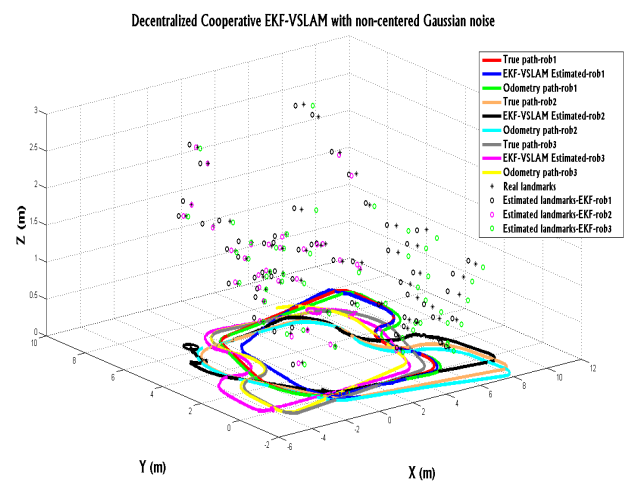

Fig. 8: Visual EKF-SLAM simulation results with noncentered Gaussian noise.

Figures 8 and 9 present the pose estimation using the cooperative EKF/ASVSF-VSLAM with non-centered Gaussian noise of the UGVs. As shown in Figures 8, 9, 10 and 11, the significant decrease of performance of the EKF-VSLAM estimator is observed when the process and observation noises are non-centered. In this case, as shown in Figures 8 and 9, the values estimated by the Cooperative ASVSF-VSLAM are less accurate than the corrected values $x, y$ and $\theta$. Decentralized Cooperative SLAM by ASVSF shows much better navigation and mapping performances than EKF-VSLAM and provides an accurate position of the UGVs. From Fig. 10 we can observe a loop closing is detected and the UGVs observe a common feature. At this moment we observe a significant improvement in the accuracy of the Adaptive SVSF-VSLAM as well as the EKF-VSLAM. The SVSF-SLAM pro-

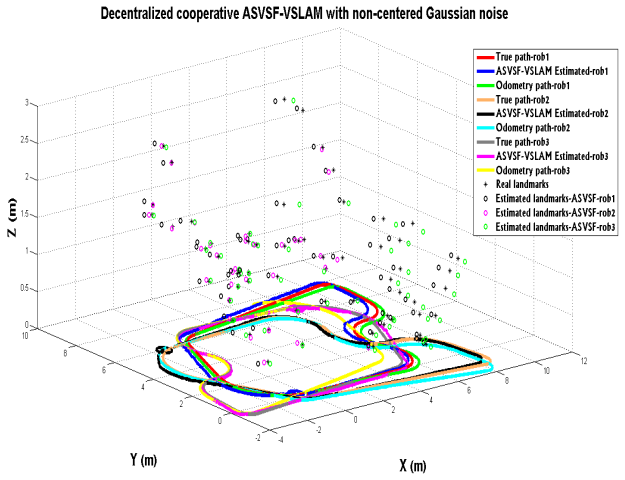

Fig. 9: Visual ASVSF-SLAM simulation results with non-centered Gaussian noise.
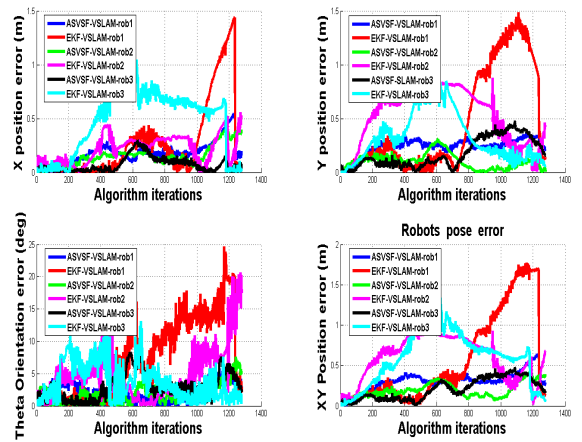

Fig. 10: Position errors of cooperative Visual SLAM with non-centered Gaussian noise.

vides the best RMSE in comparison with to the EKF-SLAM when we use non-centered Gaussian noise as shown in Fig. 11.

In the results of the first experiment, in the case of white centered Gaussian noise, the EKFSLAM algorithm gives the best results position and is more accurate than the adaptive SVSF-SLAM algorithm. This can be interpreted as follows: the system and observation models are accurate besides, when the process and observation noises are uncorrelated zero-mean Gaussian with known covariance then the EKF gives a good accuracy for position estimation. It means that the EKF-SLAM algorithm becomes the optimal filter. However, the adaptive SVSF-SLAM provide a more accurate estimate than the EKF-SLAM when we use noncentered Gaussian noise. These results clearly validate the advantage of the adaptive SVSF- 


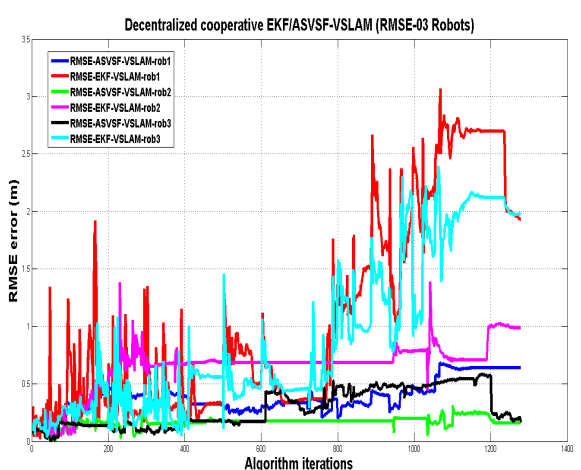

Fig. 11: RMSE Results with non-centered Gaussian noise.

SLAM over the EKF-SLAM especially when the system or observation models are not accurate enough and the process and observation noises are non-centered Gaussian noise.

\section{Conclusion}

The aim of this work is to come up with tools that are capable of producing an accurate automatic localization which could be used in an accurate map management. UGVs are a core tool in this study; we worked to improve their autonomy by solving some of their technical problems. We made an investigation of the UGVs localization, illustrated UGVs map building, and implemented a simultaneous localization and mapping solution using stereo vision sensors by two algorithms, Cooperative EKFVSLAM and Cooperative ASVSF-VSLAM. The proposed solution is extended to the Decentralized Cooperative Vision SLAM. Our proposal is a new solution for Adaptive Decentralized Cooperative SVSF-SLAM for multiple UGVs with stereo vision sensors. The adopted approach is tested with different scenarios. After validation of the proposed algorithms with simulation on three mobile robots Pioneer $3 A T$, satisfactory results (good accuracy and robustness) were obtained with adaptive SVSF Filter without any assumption on the process and/or observation model accuracy.

\section{References}

[1] Yamashita, A., Arai, T., Ota, J., \& Asama, H. (2003). Motion planning of multiple mobile robots for cooperative manipulation and transportation. IEEE Transactions on Robotics and Automation, 19, 223-237.

[2] Støy, K. (2001). Using Situated Communication in Distributed Autonomous Mobile Robotics. In SCAI, 1, 44-52.

[3] Mourikis, A.I., \& Roumeliotis, S.I. (2006). Performance Analysis of Multi-robot Cooperative Localization. IEEE Transactions on Robotics, 22(4), 666-681.

[4] Roumeliotis, S.I., \& Rekleitis, I.M. (2004). Propagation of Uncertainty in Cooperative Multi-robot Localization: Analysis and Experimental Results. Autonomous Robots, 17(1), 41-54.

[5] Burgard, W., Fox, D., \& Thrun, S. (1997). Active mobile robot localization by entropy minimization. In Advanced Mobile Robots, 1997. Proceedings., Second EUROMICRO workshop on. IEEE, 155-162.

[6] Rochaab, R., Diasa, J., \& Carvalhob, A. (2005). Cooperative multi-robot systems: A study of vision based 3D mapping using information theory. Journal of Robotics and Autonomous Systems, 53(3-4), 282-311.

[7] Martinelli, A., Pont, F., \& Siegwart, R. (2005). Multi-robot localization using relative observations (No. CONF).

[8] Trawny, N., \& Barfoot, T. (2004). Optimized motion strategies for cooperative localization of mobile robots. IEEE International Conference on Robotics and Automation, 1, 1027-1032.

[9] Ozkucur, N.E., \& Akin, H.L. (2009). Cooperative Multi-robot Map Merging Using Fast-SLAM. Robot Soccer World Cup XIII, Lecture Notes in Computer Science Springer Berlin, Heidelberg, 5949/2010, 449-460. 
[10] Dissanayake, M.G., Newman, P., Clark, S., \& Durrant-Whyte, H.F. (2001). A solution to the simultaneous localization and map building (SLAM) problem. IEEE Tran. Robot. Automat, 17(3), 229-241.

[11] Gil, A., Reinoso, I., Ballesta, M., \& Julia, M. (2010). Multi-robot visual SLAM using a Rao-Blackwellized particle filter. Robotics and Autonomous Systems, Robotics and Autonomous Systems, 58(1), 68-80.

[12] Andersson, L. A., \& Nygards, J. (2008). C-SAM: Multi-robot SLAM using square root information smoothing. In Robotics and Automation, 2008. ICRA 2008. IEEE International Conference on. IEEE, 27982805 .

[13] Gadsden, S. A., Dunne, D., Habibi, S. R., \& Kirubarajan, T. (2009). Comparison of extended and unscented Kalman, particle, and smooth variable structure filters on a bearing-only target tracking problem. In Signal and Data Processing of Small Targets 2009 (Vol. 7445, p. 74450B). International Society for Optics and Photonics.

[14] Al-Shabi, M. (2011). The General Toeplitz/Observability SVSF. Department of Mechanical Engineering, Mc Master University, Hamilton, Ontario, Ph.D.

[15] Sfeir, J. (2009). Navigation d'un Robot Mobile en Environnement Inconnu Utilisant les Champs de Potentiels Artificiels. Maïtrise en génie de la production automatisée, Ecole de Technologie Superieure, Montriall, Canada.

[16] Ahmed, A., Abdelkrim, N., \& Mustapha, H. (2016). Smooth Variable Structure Filter VSLAM. IFAC-PapersOnLine, 49(15), 205211.

[17] Yan, X., Zhao, C., \& Xiao, J. (2011). A novel FastSLAM algorithm based on iterated unscented Kalman filter. In Robotics and Biomimetics (ROBIO), 2011 IEEE International Conference on. IEEE, 19061911.
[18] Habibi, S.R. (2007). The Smooth Variable Structure Filter. IEEE proceeding, 95(5), 1026-1059.

[19] Gadsden, S. A., \& Habibi, S. R. (2010). A new form of the smooth variable structure filter with a covariance derivation. In Decision and Control (CDC), 2010 49th IEEE Conference on. IEEE, 7389-7394.

[20] Nemra, A., \& Aouf, N. (2009). Robust airborne $3 \mathrm{D}$ visual simultaneous localization and mapping with observability and consistency analysis. Journal of Intelligent and Robotic Systems, 55(4-5), 345-376.

[21] Demim, F., Nemra, A., \& Louadj, K. (2016). Robust SVSF-SLAM for Unmanned Vehicle in Unknown Environment. Journal of IFAC-Papers OnLine Science Direct, 49(21), 386-394.

[22] Nemra, A., \& Aouf, N. (2010). Robust INS/GPS sensor fusion for UAV localization using SDRE nonlinear filtering. IEEE Sensors Journal, 10(4), 789-798.

[23] Demim, F., Nemra, A., Louadj, K., Mehal, Z., Hamerlain, M., \& Bazoula, A. (2016). Simultaneous localization and mapping algorithm for unmanned ground vehicle with SVSF filter. In Modelling, Identification and Control (ICMIC), 2016 8th International Conference on. IEEE, 155-162.

[24] Demim, F., Nemra, A., Louadj, K., Hamerlain, M., \& Bazoula, A. (2016). A new approach to improve the success and solving the UGVs Cooperation for SLAM Problem, using a SVSF Filter. In Proceedings of the Mediterranean conference on pattern recognition and artificial intelligence. ACM, 5663.

[25] Demim, F., Boucheloukh, A., Nemra, A., Louadj, K., Hamerlain, M., Bazoula, A., \& Mehal, Z. (2017). A new adaptive smooth variable structure filter SLAM algorithm for unmanned vehicle. In Systems and Control (ICSC), 2017 6th International Conference on. IEEE, 6-13.

[26] Demim, F., Nemra, A., Louadj, K., Hamerlain, M., \& Bazoula, A. (2017). Cooperative 
SLAM for multiple UGVs navigation using SVSF filter. Automatika, 58(1), 119-129.

[27] Demim, F., Nemra, A., Louadj, K., Hamerlain, M., \& Bazoula, A. (2018). An adaptive SVSF-SLAM algorithm to improve the success and solving the UGVs cooperation problem. Journal of Experimental \& Theoretical Artificial Intelligence, 30(3), 389414.

[28] Demim, F., Nemra, A., Louadj, K., \& Hamerlain, M. (2018). Simultaneous localization, mapping, and path planning for unmanned vehicle using optimal control. Journal of Advances in Mechanical Engineering, 10(1), 1-25.

[29] Demim, F., Nemra, A., Louadj, K., Boucheloukh, A., Kobzili, E., Allam, A., Hamerlain, M. \& Bazoula, A. (2018). Visual SVSF-SLAM Algorithm Based On Adaptive Boundary Layer Width. (Eds.): ICEECA'2017, Book: Advanced Control Engineering Methods in Electrical Engineering Systems, SPRINGER Nature Switzerland AG.

[30] Demim, F., Nemra, A., \& Louadj, K. (2018). Path planning for unmanned ground vehicle. IEEE 5th International Conference on Control, Decision and Information Technologies (CoDIT'2018), Aristotle University of Thessaloniki, Greece, 748750 .

[31] Demim, F., Nemra, A., Abdelkadri, H., Louadj, K., Hamerlain, M., \& Bazoula, A. (2018). SLAM problem for autonomous underwater vehicle using SVSF filter. IEEE 25th International Conference on Systems, Signals and Image Processing (IWSSIP'2018), Maribor, Slovenia, 1-5.

\section{About Authors}

Fethi DEMIM received the Engineer degree in Electronics from the University of Abou-Bekr Belkaïd, Tlemcen, Algeria, in 2002 and M.S degree in control systems from the Department of Control, Polytechnic Military School,
Algeria, in 2013. He is currently pursuing his Ph.D. degree at the Polytechnic Military School, Algeria. His current research interests are autonomous navigation, optimization, path planning, unmanned vehicles, localization of ground vehicle and map building.

Abdelkrim NEMRA received the Engineer degree and M.S degree in Control systems from the Department of Control, Polytechnic Military School, Algeria, in 2004 and 2006, respectively. He received the Ph.D. degree in Control systems from the Department of Informatics and Sensors, Cranfield University, Shrivenham, UK in 2010. He received the accreditation to supervise research (HDR) in 2016. His current research interests include unmanned vehicles, localization of an aerial vehicle and map building. His publications include a number of conference and journal papers in computer vision and data fusion and book chapters in robotics.

Abdelghani BOUCHELOUKH received the Engineer degree and M.S degree in control systems from the Department of Control, Polytechnic Military School, Algeria, in 2012. $\mathrm{He}$ is currently pursuing his Ph.D. degree at the Polytechnic Military School, Algeria. His current research interests are autonomous navigation vehicle, path planning and localization.

Kahina LOUADJ is currently lecturer at the University of Bouira in the field of Informatics, mathematics and physics Laboratory (LIMPAF). She studied at the University of Tizi Ouzou. She received DES of Mathematics in 2000, Doctorate in 2012. Her main research interests are optimal control, optimization, aircraft problems.

Mustapha HAMERLAIN received his Ph.D in INSA Toulouse, France. Currently, he is the head of Robotic Division at the Center for Development of Advanced Technologies (CDTA), Baba Hassen, Algiers, Algeria. His current research interests include unmanned vehicles, localization of an aerial vehicle. His publications include a number of conference and journal papers and book chapters in robotics. 
Abdelouahab BAZOULA received the Engineer degree in control systems from the Polytechnic Military Institute, Algeria, in 1996, and the M.S and Ph.D degrees from the same institution, in 2002 and 2011 respectively. Currently, he is the head of the robotics laboratory at the Polytechnic Military Institute. His current research includes, vision and robotics. He has authored and co-authored many papers in the field of robotics.

"This is an Open Access article distributed under the terms of the Creative Commons Attribution License, which permits unrestricted use, distribution, and reproduction in any medium, provided the original work is properly cited (CC BY 4.0)." 